\title{
Optimal conditions for imaging in scanning tunneling microscopy: Experimental
}

\author{
E. Anguiano, ${ }^{\text {a) }}$ A. I. Oliva, ${ }^{\text {b) }}$ and M. Aguilar ${ }^{\mathrm{c})}$ \\ Instituto de Ciencia de Materiales (CSIC), Campus Universidad Autónoma de Madrid, 28049 Madrid, Spain
}

(Received 18 June 1998; accepted for publication 18 August 1998)

\begin{abstract}
We present a study of the behavior of the texture of surfaces imaged by scanning tunneling microscopy (STM) under different control conditions in the feedback loop. The quality and the reliability of the STM images can be described quantitatively by giving the values of two adimensional parameters that describe the measurement conditions. The analysis of the main surface texture parameters such as the rms roughness and the average wavelength shows that they have a strong dependence on the values of the parameters used in the feedback loop for imaging regardless of the visual quality of the images that can be the same. (C) 1998 American Institute of Physics. [S0034-6748(98)03311-5]
\end{abstract}

\section{INTRODUCTION}

Scanning tunneling microscopy (STM) and atomic force microscopy (AFM) are techniques widely used in the surface science area because of the capacity to give tridimensional surface information. STM, AFM, and all the modifications of these techniques are globally named scanning probe microscopy (SPM).

The surface quality of materials plays a very important role in several areas such as gas adsorption, ${ }^{1,2}$ catalysis, ${ }^{3}$ optical properties, ${ }^{4}$ physics-chemistry of wetting surfaces, ${ }^{5}$ porous materials, ${ }^{6}$ microelectronics, ${ }^{7}$ and thin films properties. $^{8,9}$ There has been a particular interest recently because of the discovery of the fractal behavior of surfaces. ${ }^{8-11}$ In all cases the SPM techniques play an important role because of their high precision measurements of the vertical information. Then, a good approximation on the real value of the surface quality will permit us to have confidence in them. Moreover, in most of the studies described above it is absolutely necessary to know with maximum precision that the vertical dimension and relative variations between different samples should be recorded with the least experimental errors possible.

Images obtained using these techniques are widely published but the information about the feedback loop conditions of how they were obtained never is given. In many works images obtained from different samples are compared without any information on the feedback conditions that, most probably, have been changed from sample to sample. In other words, image quality is determined only visually and there is a wide region of feedback conditions where the image quality is good. However, the human eye has a very poor gray scale discrimination-several orders of magnitude

a)Permanent address: Escuela Técnica Superior de Informática, Universidad Autónoma de Madrid, Cantoblanco, 28049 Madrid, Spain; electronic mail: eloy.anguiano@ii.uam.es

b) On postdoctoral stay from Cinvestav Unidad Mérida, A.P. 73 Cordemex, 97310 Mérida, Yucatán, México; electronic mail:

oliva@kin.cieamer.conacyt.mx

${ }^{c)}$ Electronic mail: maguilar@icmm.csic.es lower than the analog-to-digital (A/D) converter and display/ picture capacity. Thus, the discrimination of image quality usually used is too poor to decide the optimal conditions for an image. In a recent work published in this issue, ${ }^{12}$ we discussed the theory on the optimal conditions for the feedback loop to obtain reliable STM images. The study includes the stability, amplitude, and phase conditions that the feedback loop needs to have when images are obtained. As mentioned above, until now, the quality of SPM images is appreciated only visually; in other words, there is no care on the accuracy of the image. In this work we study, by using the surface texture parameters extended to surfaces, the problems and mistakes that will occur in the analysis of surface parameters when STM images are not obtained using good control conditions, in spite of the high image quality visually observed.

\section{SURFACE TEXTURE PARAMETERS}

The surface texture parameters used in this work are taken from the international standards BS 1134 and ISO 468. However, they are defined for single profiles and SPM techniques give three-dimensional (3D) information: $x, y$, and vertical $(z)$. Then, to be used on SPM data, it is necessary to extend the standards for profiles to surfaces. The extended relations are explained below:

For a single profile, the root mean square of roughness $R_{q}$ is defined as:

$$
R_{q}=\sqrt{\frac{1}{L} \int_{0}^{L} z^{2}(z) d x},
$$

where $z(x)$ is the value of the height in position $x$ of the profile when the mean value is subtracted, i.e., the departure from the mean line. The extended relation $R_{q}$ for surfaces is given by:

$$
R_{q}=\sqrt{\frac{1}{L_{x} L_{y}} \int_{0}^{L_{x}} \int_{0}^{L_{y}} z^{2}(x, y) d x d y .}
$$


Now, $z(x, y)$ is the surface height when the mean plane is subtracted. If the last equation is digitally acquired and it is represented with $N$ points in both directions, the equation for the $R_{q}$ value is given by

$$
R_{q}=\sqrt{\frac{1}{N^{2}} \sum_{i=1}^{N} \sum_{j=1}^{N} z_{i j}^{2}}
$$

where $z_{i j}$ is value of the height at the $i-n$th point of the $j-n$th scan.

The average wavelength $\left(\lambda_{q}\right)$ is a measure of the spacing between local peaks and valleys, taking into account their relative amplitudes and individual spatial frequencies. The generalization of the $\lambda_{q}$ is difficult because its definition implies a direction. However, we calculate its value by using the $R_{q}$ by means of the relation

$$
\lambda_{q_{u}}=\frac{2 \pi R_{q}}{\Delta_{q_{u}}}
$$

where $\mathbf{u}$ is the unitary vector that indicates the direction of analysis (we have used two unitary vectors along the $x /$ scan, and $y$ directions), and $\Delta_{q_{u}}$ is the slope of the profile throughout the assessment length.

For a surface in the case that the vector is in the $x$ direction, the relation for $\Delta_{q_{x}}$ can be calculated by

$$
\Delta_{q_{x}}=\sqrt{\frac{1}{(N-1)^{2}} \sum_{i=1}^{N} \sum_{j=1}^{N-1}\left(\phi_{i j}^{x}-\left\langle\phi_{x}\right\rangle\right)^{2}},
$$

where $\phi_{i j}^{x}$ is the value of the directional derivative of the surface in the $x$ direction, and $\left\langle\phi_{x}\right\rangle$ is the mean value of the derivative in the same direction. A discrete relation of the directional derivative in the $x$ direction is given by $\phi_{i j}^{x}$ $=z_{i+1, j}-z_{i, j}$, where $z_{i, j}$ are the heights along the profile.

\section{THE G-K DIAGRAM DESCRIPTION}

In order to clarify the different conditions used to obtain the STM images, we will briefly describe the adimensional parameters $G$ and $K$ introduced in the previous work. ${ }^{12}$ The adimensional parameters contain all the terms involved in the feedback loop in order to determine the work conditions for imaging. The usage of the parameters permits us to compare easily the conditions under which images are taken with STM of different designs. Relations for $G$ and $K$ were obtained by considering the limits imposed by the mechanical stability, the amplitude, and the phase of the signal on the measurement.

Thus, the $K$ value is defined by the relation $K$ $=G_{o} K_{1} / \omega_{s}$, where $K_{1}$ is the integration constant, $\omega_{s}$ is the sampling rate, and $G_{o}$ is the gain of the open loop given by $G_{o}=A \alpha \gamma_{0} G_{1}$. Here $A$ is the amplification factor, $\alpha$ is a constant related to the work function of the tunnel junction to and the characteristics of the logarithmic amplifier, $\gamma_{0}$ is the sensitivity of the piezoelectric, and $G_{1}$ is the gain of the proportional amplifier in series.

On the other hand, the $G$ value is defined by the relation $G=G_{o} G_{2}$, where $G_{2}$ is the gain of the proportional amplifier in parallel with the integrator in the feedback loop diagram.

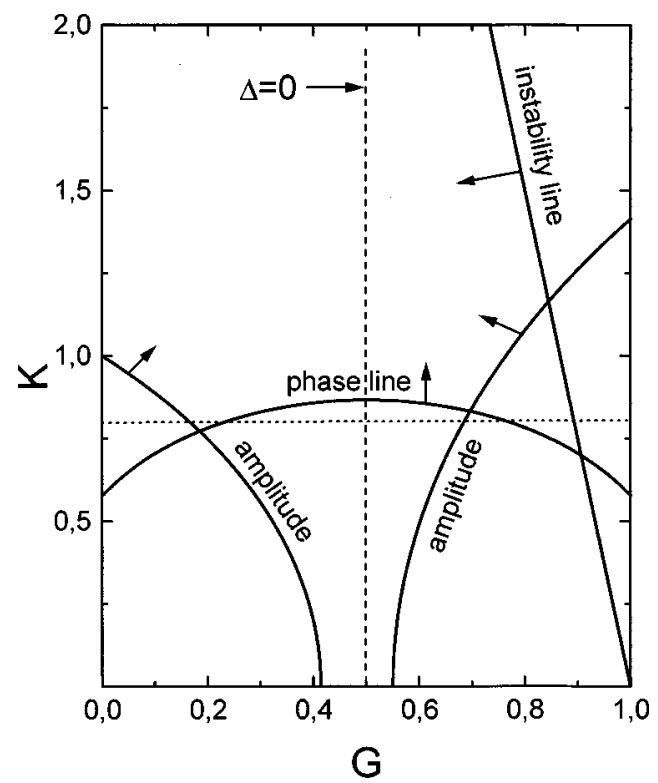

FIG. 1. The $G-K$ diagram. A group of images was taken with control conditions along the vertical line $G=0$ and the other along the horizontal line $K=0.8$.

The $G-K$ diagram is useful as a general frame to reference the conditions of all measurements of the images obtained by the SPM technique.

Figure 1 shows the $G-K$ diagram. The sloped line represents the limit for mechanical stability and depends basically on the mechanical frequency and the delay time of the SPM device. Two curved lines at the left and right of the vertical line $G=0.5$ represent the limit for the error in the amplitude $(\Delta)$ of the signal to be captured, lower than a given value. Points along the vertical dashed line $G=0.5$ means no error in the amplitude $(\Delta=0)$. The transversal curve is the limit for the value of the signal phase. Arrows on each line indicate the region for good control conditions for imaging.

By knowing the characteristics of the microscope used for imaging, we can draw the point corresponding to the particular measurement conditions into the $G-K$ diagram. Depending on the point position, we can try to move it for better conditions by using different values in the parameters. Normally, the parameters that are easy to change into the feedback loop are $K_{1}, G_{1}$, and $G_{2}$. Lines drawn in Fig. 1 represents the limits for an error amplitude of $\Delta=-0.5$ (left), $\Delta=+0.5$ (right), and for an error in phase of $\phi$ $=-60^{\circ}$. The slope of the stability line corresponds to a mechanical resonant frequency of $5 \mathrm{kHz}$ and a delay time of $0.2 \mathrm{~ms}$ in our device. ${ }^{13}$

\section{EXPERIMENT}

We used evaporated gold films of $1800 \mathrm{~nm}$ thickness for imaging because of the clean surface and easy measurement. Moreover, evaporated thin films show a granular structure that it is appropriate for our study. Images were obtained in a V-3000 STM head mounted onto a scanning electron microscope (SEM) Hitachi S-2700. The electronics to control the tip-sample junction and the data acquisition system are of 
home made design and they have been widely discussed in a previous work. ${ }^{13}$ The measured STM mechanical frequency is $5 \mathrm{kHz}$ with $0.2 \mathrm{~ms}$ as delay time. Tripod sensitivity $\gamma_{0}$ is $30 \mathrm{~nm} / \mathrm{V}$ in the $z$ direction and $90 \mathrm{~nm} / \mathrm{V}$ in the $x$ and $y$ directions. All images were obtained with $256 \times 256$ pixels $800 \times 800 \mathrm{~nm}^{2}$ in size and $1 \mathrm{kHz}$ of scan rate. Under these conditions, the transfer function presents a flat behavior and assures that all images were taken far from the resonance frequency. The integrator constant value used was $999 \mathrm{~s}^{-1}$. Vertical data were obtained with 12 bit resolution. Changes in the conditions in the feedback loop were made by only changing $G_{1}$ and $G_{2}$ gain values. We used electrochemically prepared tungsten tips. ${ }^{14}$ Given that the tip plays an important role in imaging, we diminished its effect by obtaining all the images with the same tip and into the same sample zone for the different conditions used. All images were obtained scanning along the $x$ direction.

\section{RESULTS}

The results obtained in this section are both qualitative and quantitative. Qualitative results are obtained by visual inspection of the STM images taken under different measurement conditions. Quantitative results are represented by the surface texture parameters calculated from the images. For a good representation of all the different conditions included into the $G-K$ diagram, we will analyze two cases: (a) points along a vertical line $(G=$ constant $)$ and (b) points along a horizontal line ( $K=$ constant).

\section{A. Points along a $G=$ constant line}

In this case, STM images were taken under control conditions along the line $G=0$ for different values on the $K$ parameter. These are conditions along a vertical line on the $G-K$ diagram and very far from the instability condition of the device. This case implies that the amplifier parallel to the integrator in the proportional integral (PI) feedback circuit is not present. This kind of control circuit has been used many times in the literature.

We obtained all images with the same tip and in the same sample zone going from high $K$ values down to low $K$ values to be sure that at the beginning there are better conditions for imaging than at the end. Figure 2 shows some images taken with $K$ values ranging from 1.5 to 0.2 . In our case, lower $K$ values imply a larger phase shift. We can visually observe that the images lose quality when the phase shift is larger.

Figure 3 shows the $R_{q}, \lambda_{x}$, and $\lambda_{y}$ values calculated from the images. We clearly see how the $R_{q}$ value decreases as the images are obtained with smaller $K$ values. Points labeled a, b, c, and d in Fig. 3 correspond to the corresponding images presented in Fig. 2.

A consequence of the larger phase shift reached at lower $K$ values is the increment in the average wavelengths. The most important changes occur in the scan direction $x$. The average wavelength perpendicular to the scan direction is slightly affected. The reason is clear: when the phase shift is large the effect occurs only in the scan direction because in
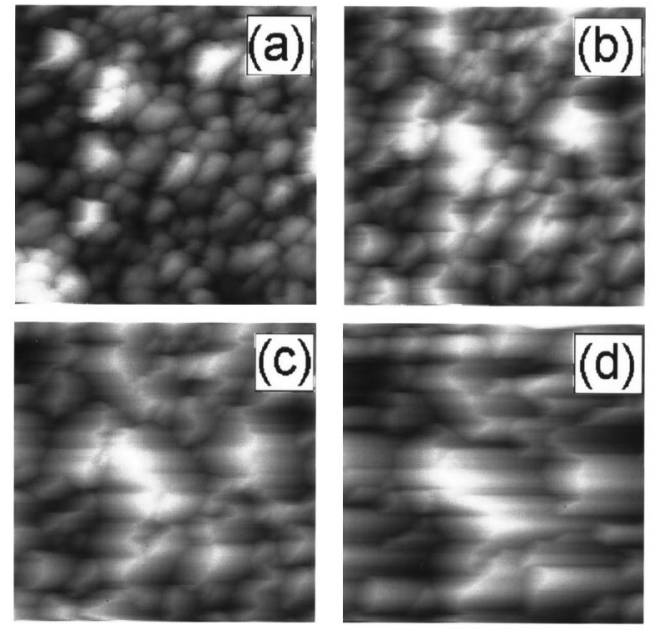

FIG. 2. Forward STM images taken under conditions along the vertical line $(G=0)$ from higher to lower $K$ value.

the orthogonal direction the time lapse between adjacent points is much greater and then there is no phase shift.

Thus, the surface texture data calculated from images are reliable when $K>1$, otherwise, amplitude and phase shift are so large that they yield erroneous results. In other words, it is possible to use a feedback circuit for control and measurement in STM that includes only an integrator if measurements are made with the condition $K>1$.

Here, it is important to mention that the tip did not touch the surface sample during imaging in this experiment and then, after arriving at the lower $K$ value, we were able to reproduce the first image taken with the higher $K$ value.

\section{B. Points along a $K=$ constant line}

Now we will study STM images obtained under different conditions along a horizontal line, i.e., the same $K$ value ( $K=0.8$ ) and different $G$ values. We choose $K=0.8$ because of the good conditions given by the $G-K$ diagram. The $G$ value used was changed from 0 to 2.5 . In this way, we represent conditions for imaging that brings us to the instability conditions given by the resonance frequency. Images obtained did not show visually important changes and for this reason they are not shown. However, the effect of the insta-

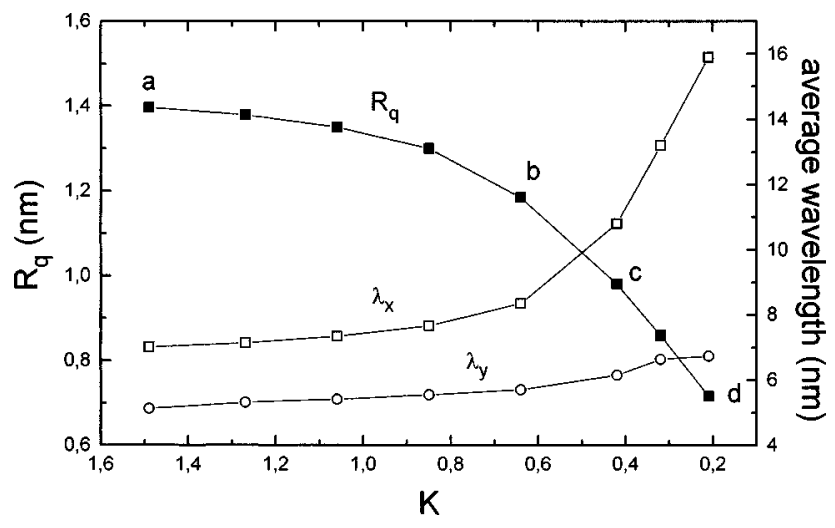

FIG. 3. Roughness $\left(R_{q}\right)$ and average wavelength $\left(\lambda_{q}\right)$ obtained as a function of the different conditions used along the $G=0$ line. (a) - (d) correspond to images showed in Fig. 2. 


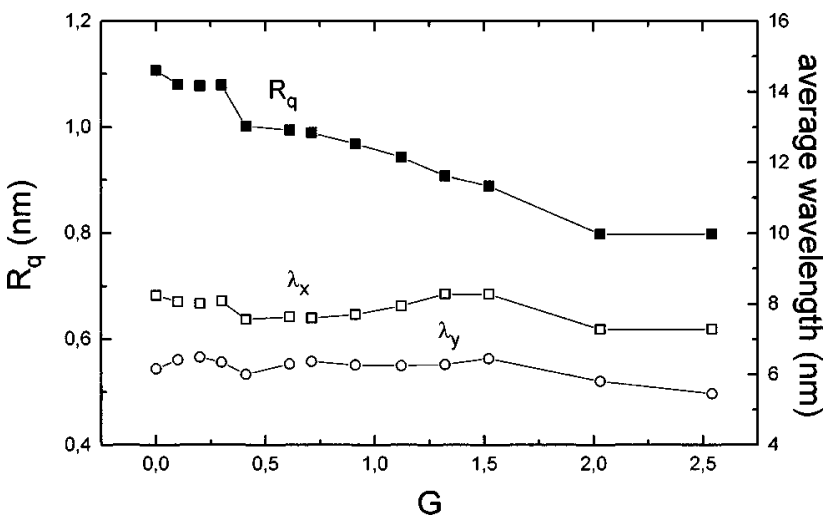

FIG. 4. Roughness $\left(R_{q}\right)$ and average wavelength $\left(\lambda_{q}\right)$ as function of the different conditions used along the $K=0.8$ line. STM images are visually similar and are not presented.

bility conditions are observed in images obtained with $G$ $>1$ because the instrument is in the instability region as a consequence of the proximity to the mechanical resonant frequency of the device. In this way, it is important to note that for the case of $G=2.5$ (far into the instability region), a resonance at high frequency was pretty clear in the image but it does not produce damage either to the tip or to the sample. Moreover, it changes the $R_{q}, \lambda_{x}$, and $\lambda_{y}$ values significantly.

Figure 4 shows the small variations on the $R_{q}, \lambda_{x}$, and $\lambda_{y}$ values as a function of the $G$ values used for imaging. The changes can be justified by the small interactions between tip and sample. The tip did not suffer damage by the resonance found in the different images. However, the increase in the signal/noise ratio on the image makes the noise observable in the images.

\section{DISCUSSION}

STM images obtained out of the optimal control conditions can provide erroneous results in the surface texture measurement than are not easy to detect visually. Under some conditions, the tip and the surface can be modified because the tip touches the sample. This will ensure that the following images are affected. STM images obtained with the same tip and under optimal conditions will always maintain a good control on the measurement conditions and a good reproducibility.
As shown in the results, the surface texture parameters can be used to measure the image's quality in a quantitative way. To work out of the good region affects in a quantitative way, the surface roughness and the other surface texture parameters of a surface measured by an STM. Then, the conclusions published on properties, models, etc. obtained from analysis of the vertical data from images taken out of good control conditions or in unknown conditions need to be revised because of the results found here.

The adimensional parameters $G$ and $K$ are essentially data that should be reported in all works on STM to assure the quality and the experimental reliability of the images. In fact, the $G$ and $K$ values used for imaging can give an idea of the quality and reliability of the images presented in the articles.

\section{ACKNOWLEDGMENTS}

One of the authors, A. I. Oliva, thanks CONACYT (México) for the grant given to him for his postdoctoral fellowship at ICMM-CSIC, Spain. This work was supported by Grant No. MAT96-2514E from CICYT, Spain.

${ }^{1}$ P. Pfeifer, D. Avnir, and D. Farin, Surf. Sci. 126, 569 (1993).

${ }^{2}$ P. Pfeifer, Y. J. Wu, M. W. Cole, and J. Krim, Phys. Rev. Lett. 62, 1997 (1989).

${ }^{3}$ P. Pfeifer and D. Avnir, J. Chem. Phys. 79, 3558 (1983).

${ }^{4}$ M. Moskovits, Rev. Mod. Phys. 57, 783 (1985).

${ }^{5}$ P. G. de Gennes, Rev. Mod. Phys. 57, 827 (1985)

${ }^{6}$ M. A. Rubio, C. A. Edwards, A. Dougherty, and J. P. Gollub, Phys. Rev. Lett. 63, 1685 (1989).

${ }^{7}$ D. W. Malone and R. E. Hummel, Crit. Rev. Solid State Mater. Sci. 22, 199 (1997).

${ }^{8}$ D. Avnir, The Fractal Approach to Heterogeneous Chemistry (Wiley, New York, 1990).

${ }^{9}$ A. L. Barabasi and H. E. Stanley, Fractal Concepts in Surface Growth (Cambridge University Press, New York, 1995).

${ }^{10}$ P. Meakin, Fractals, Scaling and Growth Far From Equilibrium (Cambridge University Press, New York, 1998).

${ }^{11}$ Dynamics of Fractal Surfaces, edited by F. Family and T. Viseck (World Scientific, Singapore 1991).

${ }^{12}$ E. Anguiano, A. I. Oliva, and M. Aguilar, Rev. Sci. Instrum. 69, 3867 (1998)

${ }^{13}$ E. Anguiano, A. I. Oliva, M. Aguilar, and J. L. Peña, Rev. Sci. Instrum. 67, 2947 (1996).

${ }^{14}$ A. I. Oliva, A. Romero G., J. L. Peña, E. Anguiano, and M. Aguilar, Rev. Sci. Instrum. 67, 1917 (1996). 